\title{
Real-world response of patients with locally advanced pancreatic adenocarcinoma to high intensity focused ultrasound treatment: a single-center, observational study in China
}

\author{
Congye Wu", Xinmei Wu", Huanyu Zhao, Dongying Gu, Xiaowei Wei, Cuiju Tang, Jinfei Chen \\ Department of Oncology, Nanjing First Hospital, Nanjing Medical University, Nanjing 210006, China \\ Contributions: (I) Conception and design: J Chen; (II) Administrative support: C Wu, J Chen; (III) Provision of study materials or patients: All \\ authors; (IV) Collection and assembly of data: H Zhao, X Wei; (V) Data analysis and interpretation: C Wu, D Gu, C Tang; (VI) Manuscript writing: \\ All authors; (VII) Final approval of manuscript: All authors. \\ \#These authors contributed equally to this work. \\ Correspondence to: Jinfei Chen. Department of Oncology, Nanjing First Hospital, Nanjing Medical University, 68, Changle Road, Nanjing 210006, \\ China. Email: goldflychen@163.com.
}

Background: The purpose of this study was to evaluate the feasibility of high intensity focused ultrasound
(HIFU) ablation for tumor response and local control in selected patients with unresectable locally advanced
pancreatic cancer (LAPC).
Methods: Forty-six malignant cases of unresectable pancreatic cancer were treated in our center from
February 2013 to December 2016, and were included in this analysis. Response was determined with the
response evaluation criteria in solid tumors (RECIST v1.1). Overall survival (OS), including 12 -month
survival rates and complications were also recorded and analyzed.
Results: Clinical responses (ablation obtained) were observed in $43.48 \%$ of the cases, and disease control
[complete response (CR), partial response (PR), and stable disease (SD)] was observed in 43 (93.48\%) of 46
cases. Stratified analyses suggested that receiving radiotherapy per-HIFU might affect the response rate.
Median OS was 9.7 months, with $95 \%$ CI, $7.9-13.2$ months. The 1-year survival rate was estimated to be
$46.69 \%$ (95\% CI, $31.71-60.34 \%$ ). Age <65 years, tumors located in the tail of pancreas, stage III, receiving
targeted therapy per-HIFU, time to HIFU from diagnose <2 months, and receiving HIFU therapy for more
than 10 times was detected to be significant associated with the improvement of median survival time (MST)
and $1-y e a r$ survival rate by univariate analysis. However, multivariate analysis did not support the association.
Major complications included fatigue [2], abdominal pain [2], fever [1], GI bleeding [1]. No deaths were
registered during the course of the treatment.

Conclusions: HIFU is a potentially effective and safe modality for the treatment of malignant tumors. HIFU proves to have a survival advantage in treating unresectable pancreatic cancer.

Keywords: High intensity focused ultrasound (HIFU); pancreatic adenocarcinoma; unresectable; response; survival

Submitted May 28, 2018. Accepted for publication Nov 05, 2018.

doi: $10.21037 /$ tcr.2018.11.20

View this article at: http://dx.doi.org/10.21037/tcr.2018.11.20

\section{Introduction}

Pancreatic cancer is one of the mostly diagnosed aggressive malignancy around the world, with poor longterm prognosis $(1,2)$. Pancreatic carcinoma patients are often in the advanced stage and unresectable at the time of diagnose, due to the difficulties in early diagnosis. Fewer treatment options would be feasible for them. By the latest report, pancreatic adenocarcinoma is the third 
cause of cancer-induced death around the world, with 5 -year overall survival (OS) rate of $8 \%$, while the survival is relative better in the localized cases with the rate of $32 \%$ (1). These patients with unresectable tumor lesions located in the celiac axis and the superior mesenteric artery without evidence of distant metastasis are diagnosed with locally advanced pancreatic cancer (LAPC). Patients with LAPC account for approximately $30 \%$ of all the cases of pancreatic carcinoma (3), with a median survival time (MST) of less than 10 months (4).

The front-line therapy options for LAPC patients are chemotherapy and radiotherapy, by which survival benefit is limited, complications and adverse events are frequency. Although some newer chemotherapy regimens like gemcitabine plus nab-paclitaxel (5), dasatinib plus gemcitabine $(6)$, FOLFOX-6 $(7,8)$ or FOLFIRINOX (9-11) appear and have shown a substantial survival benefit in patients with LAPC, the long-term prognosis is still poor and the heterogeneity between these studies is significant (12). Therefore, an optimal treatment for LAPC patients should provide survival benefit, alleviate pain, improve quality of life, but not cause severe compactions.

High intensity focused ultrasound (HIFU) is an emerging noninvasive ablation procedure which can ablate various solid tumors including LAPC. It can focus ultrasound energy on the target lesions and induces tumor coagulation necrosis by thermal effect (13). Several clinical trials of HIFU palliative therapy for pancreatic carcinoma cases have provided promising results $(14,15)$. HIFU monotherapy (16-21) or in combination with systemic chemotherapy $(22,23)$ was both proved to be able to relieve pain, and might bring an additional survival benefit with rare severe adverse events.

We designed a retrospective observational study, including the large number of patients with unresectable LAPC to date. The aim of this study was to assess the clinical benefit and safety of HIFU treatment for LAPC cases on local tumor response, quality of life and symptom intensity, in particular on relief of cancer-related pain.

\section{Methods}

\section{Patients}

A retrospective analysis was conducted of unresectable LAPC patients who underwent HIFU ablation at the Department of Oncology, Nanjing First Hospital, Nanjing, China, from February 2013 to December 2016. The inclusion criteria are as follows: patients with age $\geq 18$ years; patients with adequate hepatic, renal, and bone marrow function (white blood cell $\geq 3.9 \times 10^{9} / \mathrm{L}$, absolute neutrophil count $\geq 1.5 \times 10^{9} \mathrm{~L}$, platelets $\geq 100 \times 10^{9} / \mathrm{L}$, and hemoglobin $\geq 10 \mathrm{~g} / \mathrm{dL}$, serum creatinine $\leq 150 \mathrm{mmol} / \mathrm{L}$ ); and patients with an Eastern Cooperative Oncology Group performance status (ECOG PS) of 0-2. In addition, some cases should be excluded including pregnancy, lactation, metabolic disease, prior cerebrovascular event, active second malignancy, and uncontrolled intermittent illness.

The study was approved by the ethics committee of Nanjing First Hospital, and was done in accordance with the Declaration of Helsinki. Each patient signed a document of informed consent.

\section{Procedures}

HIFU was performed using HIFUINT-9000 system (Shanghai A\&S Sci-Tec Co., LTD, Shanghai, China), which is a US-guided device (22). Firstly, tumor location, size and morphological characteristic is identified by b-mode sonography, CT, or MRI, in the meantime, the influence of tumor on adjacent organs and blood vessels is also evaluated. Next, the detecting head of this system will complete the re-localization of the therapy area. Finally, the ablation energy focus is controlled to move along with a three-dimensional axis orderly until to cover the target lesions. The main HIFU parameters of treatment in this study were the following: input power, $5-10 \mathrm{~kW} / \mathrm{cm}^{2}$; therapy depth, $2-15 \mathrm{~cm}$; practice-focused sphere, $3 \mathrm{~mm} \times$ $3 \mathrm{~mm} \times 8 \mathrm{~mm}$; unit transmit time (t1):intermission time (t2) $=1: 2$; and HIFU times at each lesion, 8-10 times. All of the parameters can be varied depending on the depth of tumor.

\section{Observation and measurement}

The primary outcome of this study was tumor response rate. The secondary endpoints were OS, including 12-month survival rates, and safety. Tumor assessments were done with a CT or MRI scan at baseline, 1, 3, 6 and 12 months after HIFU treatment. Response was determined according to the response evaluation criteria in solid tumors (RECIST v1.1) (24). Adverse events were recorded, and the severity was graded in accordance with the CTCAE, version 4 (25).

\section{Statistical analysis}

The data are presented as the mean \pm SD for normally 
distributed data or medians for non-normally distributed data. OS analyses of patients were conducted by KaplanMeier method. For more detailed descriptions of the survival, stratified analyses by the characteristics of cases were also performed. Potential clinic pathologic factors for influencing the OS rates were evaluated by log-rank test (univariate analysis) and then Cox proportional hazards model (multivariate analysis). All the data analyses were performed by STATA 12.0 software (College Station, TX, USA). $\mathrm{P}$ value $<0.05$ indicated statistically significant.

\section{Results}

\section{Patient baseline characteristics}

Forty-six patients were finally enrolled in our study. The cases were consisted with 30 males and 16 females, with median age of 64 years (58-86 years). Median Karnofsky Performance Status (KPS) was 70, in which 37 cases $\geq 60$ and 9 cases $<60$. Numerical rating scale (NRS) was commonly scored from 4 to 6 for majority cases (26/46), followed by a score interval of 1 to 3 . A majority (36/46) of the tumors were located in the head of pancreas, and the most patients (31/46) were diagnosed as IV stage pancreatic cancer. Per-HIFU treatment, 4 patients received surgery, 28 cases received chemotherapy, including gemcitabine, cisplatin, and paclitaxel (GCP), docetaxel and cisplatin (DP), gemcitabine and cisplatin (GP), FOLFIRINOX, etc. Nineteen patients received targeted therapy (apatinib, endostar, or bevacizumab), and 10 received radiotherapy. The details of baseline characteristics of patients and tumors were described in Table 1.

Table 1 Patient baseline characteristics $(\mathrm{N}=46)$

\begin{tabular}{llc}
\hline Characteristics & Category & $\mathrm{n}(\%)$ \\
\hline Gender & Male & $30(65.22)$ \\
& Female & $16(34.78)$ \\
Age & Median [range] & $64[53-86]$ \\
& $\geq 65$ & $23(50.00)$ \\
& $<65$ & $23(50.00)$ \\
KPS & Median [range] & $70[40-80]$ \\
& $\geq 60$ & $37(80.43)$ \\
& $<60$ & $9(19.57)$ \\
Tumor location & Head & $36(78.26)$ \\
& Tail & $10(21.74)$ \\
\hline
\end{tabular}

Table 1 (continued)
Table 1 (continued)

\begin{tabular}{|c|c|c|}
\hline Characteristics & Category & n (\%) \\
\hline \multirow[t]{2}{*}{ Stage } & III & $15(32.61)$ \\
\hline & IV & $31(67.39)$ \\
\hline \multirow[t]{5}{*}{ NRS } & Median [range] & $4[1-7]$ \\
\hline & 0 & $0(0.00)$ \\
\hline & $1-3$ & $18(39.13)$ \\
\hline & $4-6$ & $26(56.52)$ \\
\hline & $7-10$ & $2(4.35)$ \\
\hline \multirow[t]{3}{*}{ CA 19-9 } & Median [range] & $\begin{array}{c}427.3[0 \text { to } \\
>10,000]\end{array}$ \\
\hline & Positive & $40(86.96)$ \\
\hline & Negative & $6(13.04)$ \\
\hline \multirow[t]{3}{*}{ HIFU times } & Median [range] & $10[5-12]$ \\
\hline & $\geq 10$ & $26(56.52)$ \\
\hline & $<10$ & $20(43.48)$ \\
\hline \multirow[t]{2}{*}{ Surgery per-HIFU } & Surgery & $4(8.70)$ \\
\hline & None & $42(91.30)$ \\
\hline \multirow{10}{*}{ Chemotherapy per-HIFU } & GCP & $2(4.35)$ \\
\hline & DP & $2(4.35)$ \\
\hline & EP + GEMOX + octreotide & $2(4.35)$ \\
\hline & FOLFIRINOX & $2(4.35)$ \\
\hline & GP & $5(10.87)$ \\
\hline & GP + FOLFIRINOX & $2(4.35)$ \\
\hline & GS1 & $5(10.87)$ \\
\hline & S1 & $4(8.70)$ \\
\hline & Capecitabine & $4(8.70)$ \\
\hline & None & $18(39.13)$ \\
\hline \multirow{4}{*}{$\begin{array}{l}\text { Targeted therapy per- } \\
\text { HIFU }\end{array}$} & Apatinib & $5(10.87)$ \\
\hline & Endostar & $12(26.09)$ \\
\hline & Endostar+ bevacizumab & $2(4.35)$ \\
\hline & None & $27(58.70)$ \\
\hline \multirow[t]{2}{*}{ Radiotherapy per-HIFU } & Radiotherapy & $10(21.74)$ \\
\hline & None & $36(78.26)$ \\
\hline \multirow{3}{*}{$\begin{array}{l}\text { Time to HIFU from diag- } \\
\text { nose }\end{array}$} & Median [range] & $2[1-16]$ \\
\hline & $<2$ & $26(56.52)$ \\
\hline & $\geq 2$ & $20(43.48)$ \\
\hline
\end{tabular}

KPS, Karnofsky Performance Status; NRS, numerical rating scale; HIFU, high intensity focused ultrasound; GCP, gemcitabine + cisplatin + paclitaxel; DP, docetaxel + cisplatin; GP, gemcitabine + cisplatin; EP, etoposide + cisplatin; GS1, gemxitabine + S1. 
Table 2 RECIST response and subgroup analysis

\begin{tabular}{|c|c|c|c|c|c|c|c|}
\hline Characteristics & Category & $\mathrm{CR}$ & PR & SD & PD & $\chi^{2}$ & $\mathrm{P}$ \\
\hline \multirow[t]{2}{*}{ Gender } & Male & 3 & 8 & 16 & 3 & 2.81 & 0.422 \\
\hline & Female & 3 & 6 & 7 & 0 & & \\
\hline Age & $\geq 65$ & 1 & 8 & 12 & 2 & 3.33 & 0.344 \\
\hline \multirow[t]{2}{*}{ Tumor location } & Head & 3 & 10 & 20 & 3 & 5.06 & 0.168 \\
\hline & Tail & 3 & 4 & 3 & 0 & & \\
\hline \multirow[t]{2}{*}{ Stage } & III & 4 & 5 & 5 & 1 & 4.47 & 0.215 \\
\hline & IV & 2 & 9 & 18 & 2 & & \\
\hline \multirow[t]{2}{*}{ Surgery per-HIFU } & Surgery & 0 & 1 & 20 & 0 & 1.45 & 0.694 \\
\hline & None & 6 & 13 & 3 & 3 & & \\
\hline \multirow[t]{2}{*}{ Chemotherapy per-HIFU } & Chemotherapy & 5 & 10 & 12 & 1 & 3.61 & 0.307 \\
\hline & None & 1 & 4 & 11 & 2 & & \\
\hline \multirow[t]{2}{*}{ Targeted therapy per-HIFU } & Targeted therapy & 5 & 5 & 7 & 2 & 6.47 & 0.091 \\
\hline & None & 1 & 9 & 16 & 1 & & \\
\hline \multirow[t]{2}{*}{ Radiotherapy per-HIFU } & Radiotherapy & 1 & 2 & 4 & 0 & 11.6 & 0.009 \\
\hline & None & 5 & 12 & 19 & 3 & & \\
\hline \multirow{2}{*}{ NRS } & $4-6$ & 3 & 8 & 15 & 0 & & \\
\hline & $7-10$ & 0 & 0 & 1 & 1 & & \\
\hline \multirow[t]{2}{*}{ HIFU times } & $\geq 10$ & 6 & 9 & 10 & 1 & 7.21 & 0.066 \\
\hline & $<10$ & 0 & 5 & 13 & 2 & & \\
\hline
\end{tabular}

KPS, Karnofsky Performance Status; NRS, numerical rating scale; CR, complete response; PR, partial response; SD, stable disease; PD, progression disease; RECIST, response evaluation criteria in solid tumors; HIFU, high intensity focused ultrasound.

\section{Clinical response}

Complete response (CR), partial response (PR), stable disease (SD) and progression disease (PD) were observed in 6, 14, 23 and 3 in 46 patients administrated with HIFU ablation, respectively. Objective response rate (ORR, CR + PR) was $43.48 \%$ (20/46). Disease control rate (DCR, CR +
$\mathrm{PR}+\mathrm{SD}$ ) was $93.48 \%(43 / 46)$ (Table 2).

We conducted stratified analyses of response status by patients and tumors characteristics, finding that receiving radiotherapy per-HIFU might affect the response rate, while other factors did not reveal any influence on objective response. 
Table 3 Patients overall survival and the risk factors analysis of OS

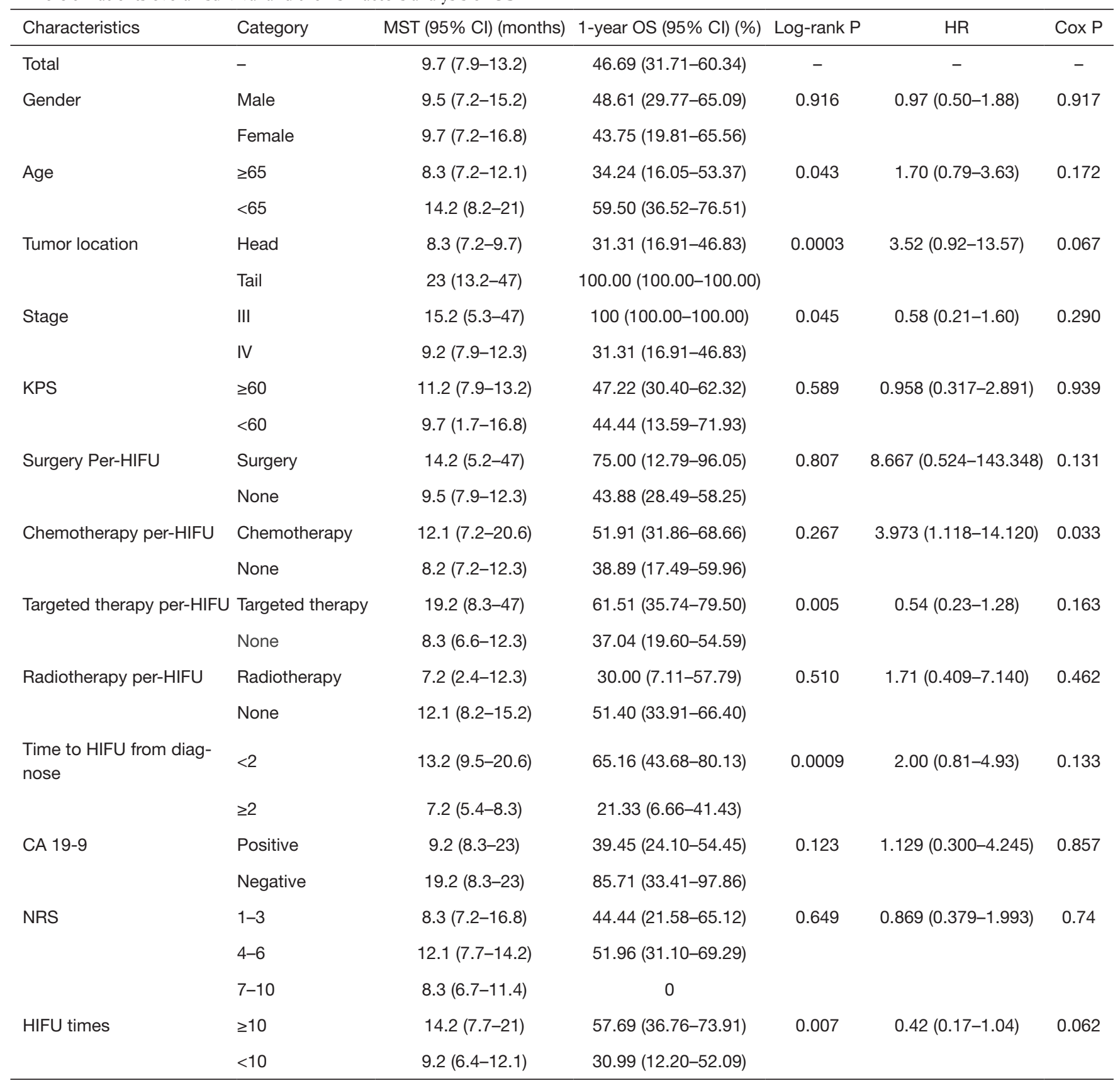

KPS, Karnofsky Performance Status; NRS, numerical rating scale; MST, median survival time; HR, hazard ratio; OS, overall survival; HIFU, high intensity focused ultrasound.

\section{OS and 12-month survival rate}

Median OS was 9.7 months, with 95\% CI, 7.9-13.2 months. The 1 -year survival rate was estimated to be $46.69 \%$ (95\% CI, 31.71-60.34\%). Stratified analyses of MST and 1 -year survival rate by gender, age, tumor location and other factors were performed to illuminate this issue in details, besides, log-rank test were also conducted. As presented in Table 3, age $<65$ years, tumors located in the tail of pancreas, stage III, receiving targeted therapy per-HIFU, time to HIFU from diagnose $<2$ months, and receiving HIFU therapy for more than 10 times was significant associated 


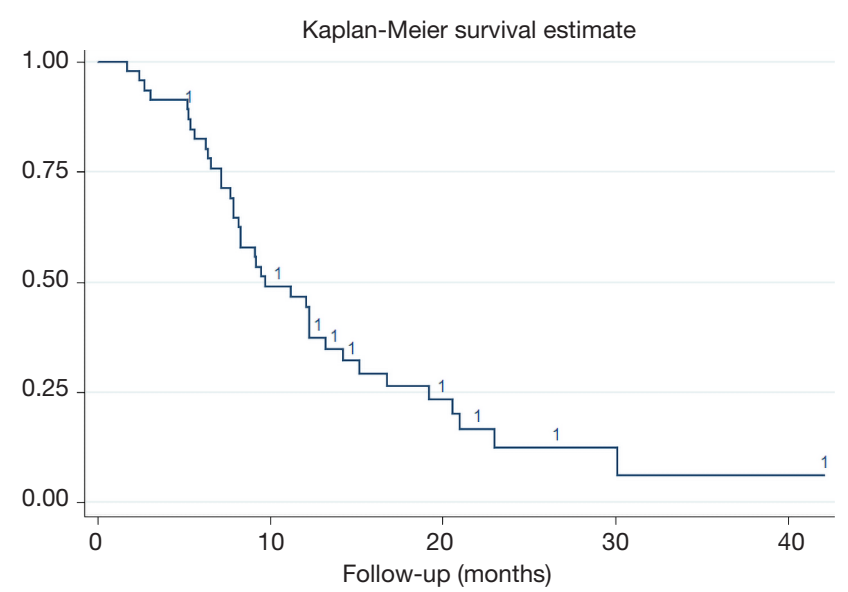

Figure 1 Kaplan-Meier survival curve of all the 46 patients.

Table 4 Most frequent adverse events

\begin{tabular}{lcc}
\hline AEs & $\mathrm{n}$ & $\%$ \\
\hline Fatigue & 2 & 4.35 \\
Abdominal pain & 2 & 4.35 \\
Fever & 1 & 2.17 \\
Gl bleeding & 1 & 2.17 \\
Total & 6 & 13.04 \\
\hline
\end{tabular}

AEs, adverse events; Gl, gastrointestinal.

with the improvement of MST and 1-year survival rate (log-rank $\mathrm{P}=0.043,0.0003,0.045,0.005,0.0009$, and 0.007, respectively). However, multivariate analysis with Cox proportional hazard regression model did not reveal any increased risk caused by patients' characteristics (Figure 1).

\section{Adverse events}

All the reported AEs were listed in Table 4. Very few cases reported complications included fatigue [2], abdominal pain [2], fever [1], GI bleeding [1]. What should be mentioned is that no skin burns were complained by any patient in our study. There was no occurrence of acute pancreatitis or peritonitis post-HIFU treatment during the followup period. Moreover, no case withdrew from this research because of the side effects and no treatment-induced death was found.

\section{Discussion}

Primary pancreatic cancer is often unresectable at the time of diagnosis and has a poor long-term prognosis, notwithstanding recent advances in chemotherapy and radiation therapy (26). Although percutaneous ablation methods have been successfully applied for the treatment of hepatic and renal tumors and are now clinically acceptable alternatives to surgery in selected patients (27), similar procedures are not yet considered by current treatment guidelines for pancreatic cancer (28), possibly because of the significant risk for massive pancreatitis or collateral damage to neurovascular structures $(29,30)$,

Ablation with HIFU is a non-invasive procedure $(16,31)$ based on the principle that focused ultrasound (US) beams cause coagulation necrosis in the target. Multiple retrospective non-randomized studies and case series on US-guided HIFU for pancreatic cancer have been reported to date, nearly all coming from Asia, especially China $(19,20,22,32)$.

As a golden standard treatment for LAPC patients, firstline treated with gemcitabine, were reported to have a median OS between 5.6 and 9.2 months in numerous high quality randomized controlled trials (33-39). HIFU treatment might prolong survival of patients, with a great improvement comparing with gemcitabine monotherapy. The 1-year OS rate of $46.69 \%$ in this study is comparable with chemotherapy and radiotherapy in LAPC $(23,40)$. In a study by Zhao et al. (41) in 2017 , the 6 -month OS was $44.4 \%$ to $100 \%$, and 12 -month OS was $11.1 \%$ to $35.4 \%$ by HIFU treatment, which is also numerical inferior to our results. Some factors should be considered, including sample size, statistical power, and baseline to be contribute to this difference.

Stratified analyses were performed to illustrate the survival status of HIFU treatment in details and aim to identify the subgroups who would obtain more clinical benefits form HIFU. Similar analyses were not described in other reports with same theme $(15,22,23,40-44)$. Our results suggested that age $<65$ years, tumors located in the tail of pancreas, stage III, receiving targeted therapy per-HIFU, time to HIFU from diagnose $<2$ months, and receiving HIFU therapy for more than 10 times might associated with the improvement of MST and 1-year survival rate. This might be significant in clinical practice when we choice a patient though multivariate analysis did not support these potential association.

In terms of the adverse events, during and after HIFU therapy, no serious adverse reactions were observed by us. Treatment-related complication as well as treatment cycle was significantly reduced compared with chemotherapy or radiotherapy $(45,46)$. Meanwhile, recent reports have 
reported certain HIFU-related complications, especially skin burn. But our results did not reveal any evidence of skin burn complication. The might be caused by the difference of HIFU equipment. As far as our knowledge goes, the device of HIFUNIT-9000 applied in our clinical practice, adopts dual focus mode, the energy upon the skin could be reduced effectively during operation comparing with other equipment.

Several limitations of this study should be acknowledged. First, our results came from a single center, non-blinded observation study, which would not be highest quality evidence of clinical practice. Second, our sample scale is relatively small, and the follow-up period is relatively short. Therefore, top-level designed trails with a larger sample size is needed. Nevertheless, our investigation has provided a reliable clinical evidence for the new direction of LAPC ablation treatment.

\section{Conclusions}

In conclusion, our encouraging results further confirmed the efficacy and safety of HIFU treatment for patients with LAPC. HIFU might be one of the optimal therapies for LAPC. However, further well-designed double-blind, randomized controlled trials are wanted to evaluate the clinical efficacy of HIFU ablation, especially in combination with chemotherapy or radiotherapy.

\section{Acknowledgments}

Funding: This work was supported by Science and Technology Development Fund of Nanjing Medical University (2017NJMUZD102).

\section{Footnote}

Conflicts of Interest: All authors have completed the ICMJE uniform disclosure form (available at http://dx.doi. org/10.21037/tcr.2018.11.20). The authors have no conflicts of interest to declare.

Ethical Statement: The authors are accountable for all aspects of the work in ensuring that questions related to the accuracy or integrity of any part of the work are appropriately investigated and resolved. The study was approved by the ethics committee of Nanjing First Hospital, and was done in accordance with the Declaration of Helsinki(as revised in 2013). All procedures performed in studies involving human participants were in accordance with the ethical standards of the Nanjing First Hospital (No. NJMU-201301-041). Informed consent was obtained from all individual participants included in the study.

Open Access Statement: This is an Open Access article distributed in accordance with the Creative Commons Attribution-NonCommercial-NoDerivs 4.0 International License (CC BY-NC-ND 4.0), which permits the noncommercial replication and distribution of the article with the strict proviso that no changes or edits are made and the original work is properly cited (including links to both the formal publication through the relevant DOI and the license). See: https://creativecommons.org/licenses/by-nc-nd/4.0/.

\section{References}

1. Siegel RL, Miller KD, Jemal A. Cancer statistics, 2018. CA Cancer J Clin 2018;68:7-30.

2. Chen $W$, Zheng R, Baade PD, et al. Cancer statistics in China, 2015. CA Cancer J Clin 2016;66:115-32.

3. Heestand GM, Murphy JD, Lowy AM. Approach to patients with pancreatic cancer without detectable metastases. J Clin Oncol 2015;33:1770-8.

4. Hsueh CT. Pancreatic cancer: current standards, research updates and future directions. J Gastrointest Oncol 2011;2:123-5.

5. Yamada S, Fujii T, Yokoyama Y, et al. Phase I study of chemoradiotherapy using gemcitabine plus nab-paclitaxel for unresectable locally advanced pancreatic cancer. Cancer Chemother Pharmacol 2018;81:815-21.

6. Evans TRJ, Van Cutsem E, Moore MJ, et al. Phase 2 placebo-controlled, double-blind trial of dasatinib added to gemcitabine for patients with locally-advanced pancreatic cancer. Ann Oncol 2017;28:354-61.

7. Ghosn M, Farhat F, Kattan J, et al. FOLFOX-6 combination as the first-line treatment of locally advanced and/or metastatic pancreatic cancer. Am J Clin Oncol 2007;30:15-20.

8. Ghosn M, Saroufim A, Kattan J, et al. Sequential FOLFOX-6 and gemcitabine for locally advanced and/or metastatic pancreatic cancer. Med Oncol 2012;29:2831-7.

9. Lakatos G, Petranyi A, Szucs A, et al. Efficacy and Safety of FOLFIRINOX in Locally Advanced Pancreatic Cancer. A Single Center Experience. Pathol Oncol Res 2017;23:753-9.

10. Suker M, Beumer BR, Sadot E, et al. FOLFIRINOX for locally advanced pancreatic cancer: a systematic review and 
patient-level meta-analysis. Lancet Oncol 2016;17:801-10.

11. Nywening TM, Wang-Gillam A, Sanford DE, et al. Targeting tumour-associated macrophages with CCR2 inhibition in combination with FOLFIRINOX in patients with borderline resectable and locally advanced pancreatic cancer: a single-centre, open-label, dose-finding, nonrandomised, phase 1b trial. Lancet Oncol 2016;17:651-62.

12. Sultana A, Smith CT, Cunningham D, et al. Meta-analyses of chemotherapy for locally advanced and metastatic pancreatic cancer. J Clin Oncol 2007;25:2607-15.

13. Orsi F, Arnone P, Chen W, et al. High intensity focused ultrasound ablation: a new therapeutic option for solid tumors. J Cancer Res Ther 2010;6:414-20.

14. Zhou Y. High-intensity focused ultrasound treatment for advanced pancreatic cancer. Gastroenterol Res Pract 2014;2014:205325.

15. Wu F. High intensity focused ultrasound: a noninvasive therapy for locally advanced pancreatic cancer. World J Gastroenterol 2014;20:16480-8.

16. Orsi F, Zhang L, Arnone P, et al. High-intensity focused ultrasound ablation: effective and safe therapy for solid tumors in difficult locations. AJR Am J Roentgenol 2010;195:W245-52.

17. Wu F, Wang ZB, Zhu H, et al. Feasibility of US-guided high-intensity focused ultrasound treatment in patients with advanced pancreatic cancer: initial experience. Radiology 2005;236:1034-40.

18. Wang K, Chen Z, Meng Z, et al. Analgesic effect of high intensity focused ultrasound therapy for unresectable pancreatic cancer. Int J Hyperthermia 2011;27:101-7.

19. Sung HY, Jung SE, Cho SH, et al. Long-term outcome of high-intensity focused ultrasound in advanced pancreatic cancer. Pancreas 2011;40:1080-6.

20. Wang K, Zhu H, Meng Z, et al. Safety evaluation of highintensity focused ultrasound in patients with pancreatic cancer. Onkologie 2013;36:88-92.

21. Gao HF, Wang K, Meng ZQ, et al. High intensity focused ultrasound treatment for patients with local advanced pancreatic cancer. Hepatogastroenterology 2013;60:1906-10.

22. Zhao H, Yang G, Wang D, et al. Concurrent gemcitabine and high-intensity focused ultrasound therapy in patients with locally advanced pancreatic cancer. Anticancer Drugs 2010;21:447-52

23. Li YJ, Huang GL, Sun XL, et al. The combination therapy of high-intensity focused ultrasound with radiotherapy in locally advanced pancreatic carcinoma. World J Surg Oncol 2016;14:60.
24. Therasse P, Arbuck SG, Eisenhauer EA, et al. New guidelines to evaluate the response to treatment in solid tumors. European Organization for Research and Treatment of Cancer, National Cancer Institute of the United States, National Cancer Institute of Canada. J Natl Cancer Inst 2000;92:205-16.

25. Liu YJ, Zhu GP, Guan XY. Comparison of the NCICTCAE version 4.0 and version 3.0 in assessing chemoradiation-induced oral mucositis for locally advanced nasopharyngeal carcinoma. Oral Oncol 2012;48:554-9.

26. Wilkowski R, Wolf M, Heinemann V. Primary advanced unresectable pancreatic cancer. Recent Results Cancer Res 2008;177:79-93.

27. Ahmed M, Brace CL, Lee FT Jr, et al. Principles of and advances in percutaneous ablation. Radiology 2011;258:351-69.

28. Tempero MA, Arnoletti JP, Behrman S, et al. Pancreatic adenocarcinoma. J Natl Compr Canc Netw 2010;8:972-1017.

29. Meijnen P, Rutgers EJ. Re: Sakorafas GH, Geraghty J, Pavlakis G. The clinical significance of axillary lymph node micrometastases in breast cancer. Eur J Surg Oncol 2004;30(8):807-16. Eur J Surg Oncol 2005;31:927.

30. Marrocchio C, Dababou S, Catalano C, et al. Nonoperative Ablation of Pancreatic Neoplasms. Surg Clin North Am 2018;98:127-40.

31. Zhou YF. High intensity focused ultrasound in clinical tumor ablation. World J Clin Oncol 2011;2:8-27.

32. Wang X, Sun J. High-intensity focused ultrasound in patients with late-stage pancreatic carcinoma. Chin Med J (Engl) 2002;115:1332-5.

33. Spano JP, Chodkiewicz C, Maurel J, et al. Efficacy of gemcitabine plus axitinib compared with gemcitabine alone in patients with advanced pancreatic cancer: an open-label randomised phase II study. Lancet 2008;371:2101-8.

34. Borad MJ, Reddy SG, Bahary N, et al. Randomized Phase II Trial of Gemcitabine Plus TH-302 Versus Gemcitabine in Patients With Advanced Pancreatic Cancer. J Clin Oncol 2015;33:1475-81.

35. Cunningham D, Chau I, Stocken DD, et al. Phase III randomized comparison of gemcitabine versus gemcitabine plus capecitabine in patients with advanced pancreatic cancer. J Clin Oncol 2009;27:5513-8.

36. Loehrer PJ Sr, Feng Y, Cardenes H, et al. Gemcitabine alone versus gemcitabine plus radiotherapy in patients with locally advanced pancreatic cancer: an Eastern Cooperative Oncology Group trial. J Clin Oncol 2011;29:4105-12.

37. Colucci G, Labianca R, Di Costanzo F, et al. Randomized 
phase III trial of gemcitabine plus cisplatin compared with single-agent gemcitabine as first-line treatment of patients with advanced pancreatic cancer: the GIP-1 study. J Clin Oncol 2010;28:1645-51.

38. Goncalves A, Gilabert M, Francois E, et al. BAYPAN study: a double-blind phase III randomized trial comparing gemcitabine plus sorafenib and gemcitabine plus placebo in patients with advanced pancreatic cancer. Ann Oncol 2012;23:2799-805.

39. New insights into the clinical profile of norfloxacin. Proceedings of a symposium held at the 6th Mediterranean Congress of Chemotherapy. Taormina, Italy, May 22-27, 1988. Eur Urol 1990;17 Suppl 1:1-51.

40. Marinova M, Rauch M, Mucke M, et al. High-intensity focused ultrasound (HIFU) for pancreatic carcinoma: evaluation of feasibility, reduction of tumour volume and pain intensity. Eur Radiol 2016;26:4047-56.

41. Zhao J, Zhao F, Shi Y, et al. The efficacy of a new high intensity focused ultrasound therapy for locally advanced pancreatic cancer. J Cancer Res Clin Oncol 2017;143:2105-11.

42. Shi Y, Ying X, Hu X, et al. Pain management of pancreatic cancer patients with high-intensity focused ultrasound therapy. Pak J Pharm Sci 2017;30:303-7.

43. Strunk HM, Henseler J, Rauch M, et al. Clinical Use of High-Intensity Focused Ultrasound (HIFU) for Tumor and Pain Reduction in Advanced Pancreatic Cancer. Rofo 2016;188:662-70.

44. Anzidei M, Marincola BC, Bezzi M, et al. Magnetic resonance-guided high-intensity focused ultrasound treatment of locally advanced pancreatic adenocarcinoma: preliminary experience for pain palliation and local tumor control. Invest Radiol 2014;49:759-65.

45. Moureau-Zabotto L, Phelip JM, Afchain P, et al. Concomitant administration of weekly oxaliplatin, fluorouracil continuous infusion, and radiotherapy after 2 months of gemcitabine and oxaliplatin induction in patients with locally advanced pancreatic cancer: a Groupe Coordinateur Multidisciplinaire en Oncologie phase II study. J Clin Oncol 2008;26:1080-5.

46. Mahadevan A, Jain S, Goldstein M, et al. Stereotactic body radiotherapy and gemcitabine for locally advanced pancreatic cancer. Int J Radiat Oncol Biol Phys 2010;78:735-42.
Cite this article as: $\mathrm{Wu} \mathrm{C}, \mathrm{Wu} \mathrm{X}$, Zhao $\mathrm{H}, \mathrm{Gu} \mathrm{D}, \mathrm{Wei} \mathrm{X}$, Tang C, Chen J. Real-world response of patients with locally advanced pancreatic adenocarcinoma to high intensity focused ultrasound treatment: a single-center, observational study in China. Transl Cancer Res 2018;7(6):1518-1526. doi: 10.21037/ tcr.2018.11.20 